\title{
Enzymatic Histo and Cytochemical Investigation on the Cardiovascular System in Spontaneously Hypertensive Rats
}

\author{
Shigeru Amano, * Fumitada Hazama, * Eiji Yamada, ${ }^{*}$ \\ and Junya Hanakita**
}

It has become clear that increased vascular permeability in spontaneously hypertensive rats (SHR) plays an important role as an intiating factor in hypertensive vascular lesions (Amanoss: J Path 121: 119, 1977, Hazama F et al : Acta Neuropath 37 : 231, 1977, Hazama $F$ et al : Advances in Neurology 20:359, 1978).

Enzymatic alteration of membrane-bound phosphatase, adenosine triphosphatase (ATPase), alkaline phosphatase (Al-Pase), 5'-nucleotidase (5'-Nucl), and lysozomal enzyme, acid phosphatase (Acid-Pase), was investigated histo and cytochemically to clarify the functional changes of the cells of vascular wall cells in SHR.

Material and Methods:

Male SHR in $\mathrm{A}_{3}$ substrain and male rats of Wistar Kyoto strain as the control animal with various ages (4-44 weeks) were used. The brain, heart, kidney, and thoratic aorta were frozen with acetone-dry ice soon after decapitation, then sliced at $8 / 1$ using a cryostat. The enzymatic reactions were as follows: ATPase and 5'-Nucl by Wachstein-Meisel procedure, Al-Pase by method of Gomori, and AcidPase by technique of Barka-Anderson. Hematoxylin and eosin staining was also done on some of the frozen sections. Wachstein-Meisel procedure was applied for electron microscopic observation of ATPase.

Result and Comments:

1) ATPase: ATPase reaction was positive in the plasma membrane and cytoplasm of the endothelial cell, medial muscle cell, and adventitial cell of control rats and young SHR. Activity in the media was increased with age in SHR, but slight or no reactive cells were also scattered about. There was a positive reaction in the proliferating cells in the thickened artery in SHR.

On electron microscopic investigation of SHR, intensive positive cells were scattered in the endothelial cell line and in the medial zone. Reaction product was detected clearly in the microvilli and pinocytotic vesicles of the endothelial cells, and in the plasma membrane and cytoplasm of the muscle cells.

Enhanced activity in the plasma membrane, pinocytotic vesicle, and microvilli is probably related to increase in the transport mecranism while the increased reaction in the cytoplasm of the muscle cell may indicate an increased contractility.

2) Al-Pase: The positive reaction was normally seen in the adventitia of the arterial tree and capillaries. Adventitial reaction was increased in SHR at 8 weeks of age. When the blood pressure had stabilized, positive reaction in the

From the Department of Pathology,* Shiga University of Medical Science, School of Medicine, Otsu; Department of Neurosurgery,** Faculty of Medicine, Kyoto University, Kyoto. 
plasma membrane and cytoplasm appeared frequently in the endothelial cells, particularly in the branching area or the thickened intima. Activity in the plasma membrane also appeared to be related to the increaseed transport function of the endcthelial cells. In case of cerebral softening or infarction of the kidney in old SHR, the enzymatic reaction had disappeared focally.

3) 5'-Nucl: The activity was positive in the adventitia of the artery, the wall of large veins and the heart capillaries in both WK and young SHR. Positive reactions were to some extent noted in the plasma membrane of muscle cells in the early stage, granular reaction in the cytoplasm of the proliferating cells as well as plasma membrane was seen segmentally. Positive activity was also present in the wall of thickened intima and in the endothelial cells. In cerebral malacia, the thickened wall of the artery and proliferated glial cells showed a positive reaction. Strongly positive activity was also seen in the cells of hypertrophic glomerulus. On the other hand, the activity was decreased in the area of sclerotic fibrosis and the adventitia of the heart, such being more evident with aging. The strong granular reaction may be an indication of increased membrane synthesis, as synthesis of plasma membrane and pinocytitic vesicles was increased in the endothelial and medial muscle cells in SHR. These appears to be a relationship between 5'-Nucl activity and cell proliferation. The activity in the proliferated cells support this theory.

4) Acid-Pase: The reaction was negative in the arterial wall in young animals and slight activity appeared mainly in the adventitia with aging in control rats. Positive reaction was seen in the cytoplasm of the medial cell, especially in the branching area or in the proliferting cells in the thickened artery in aged SHR. This reaction was present not only in the muscle cell but also in the thickened intima and the endothelial cell. Moderate reaction was detected in the pericytes and slight reaction in the muscle and endothelial cells of the intracerebral arteriole.

These increased activities indicate an enhanced function of digestion of the material which insudates into the arterial wall due to increased vascular permeability and of the damaged organelles.

\section{Summary:}

The functional abnormalities of the cells in the cardiovascular system in SHR were revealed by enzyme histo-cytochemical study. The enzymatic activity was usually increased in the area where the arteries branched and in proliferting cells in the thickened wall. Such was more remarkable in the distorted arteries with aging.

Functional roles of these 4 enzymes were discussed. 